\title{
Computing Energy Levels by Inversion of Imaginary-Time Cross-Correlation Functions ${ }^{\dagger}$
}

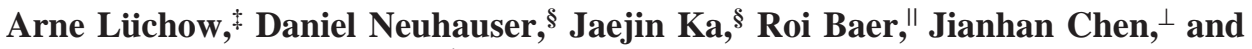

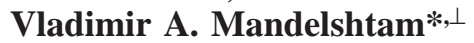 \\ Institut für Theoretische Chemie, Heinrich-Heine-Universität Düsseldorf, 40225 Düsseldorf, Germany, \\ Chemistry Department, University of California, Los Angeles, California 90095, Chemistry Department, \\ Hebrew University of Jerusalem, Givat Ram, Jerusalem, Israel, and Chemistry Department, University of \\ California, Irvine, California 92697
}

Received: February 13, 2003; In Final Form: April 14, 2003

\begin{abstract}
As proposed by Ceperley and Bernu, the excited-state energies can be obtained from imaginary-time crosscorrelation functions (rather than autocorrelation functions) generated by quantum Monte Carlo (QMC) simulations. We show that, when processed by the filter diagonalization method (FDM), the same crosscorrelation functions yield excited-state energies of higher accuracy and greater stability. The reason is that unlike the other methods FDM uses all the time domain information available. The superior performance of the cross-correlation FDM is demonstrated for a two-dimensional harmonic oscillator with closely lying eigenvalues. Because QMC does not take advantage of the separability in the Hamiltonian, this model system provides a challenging and generic test case.
\end{abstract}

\section{Introduction}

Most applications of the quantum Monte Carlo (QMC) method $^{1-3}$ are ground-state calculations where the ground state is projected with a random walk, but it is well-known that excited states can be obtained as well. If the nodes of the excited states are known, individual excited states can be calculated with the fixed-node approximation. ${ }^{4}$ An early application was the tunneling splitting in $(\mathrm{HF})_{2} .^{5}$ Later developments involved optimization of the excited-state nodes. ${ }^{6,7}$ Several studies have appeared recently where fixed-node-based methods were used to calculate excited states for electronic systems. ${ }^{8,9}$

The general alternative to the fixed-node approach is to extract the excited states from the decay of the initial state-or initial states - to the ground state. In this case, many excited states can be obtained simultaneously and-in principle-exactly within the statistical error. In this approach the ground state and excited states are obtained from the part of the quantum simulation that is usually discarded in ground-state QMC applications, i.e., by using the data from a short propagation in imaginary time $\beta$. Several such methods have been developed for extracting excited-state information from the random walk. Most widely used are the correlation function quantum Monte Carlo method (CFQMC) by Ceperley and Bernu ${ }^{10,11}$ and the projection operator imaginary time spectral evolution method (POITSE) by Blume, Lewerenz, and Whaley. ${ }^{12,13}$ The CFQMC approach has recently been used for calculating rovibrational levels; ${ }^{14-17}$ the POITSE method has been successfully applied to problems such as tunneling splittings ${ }^{18}$ and quantum structure in helium clusters. ${ }^{19-23}$

In this paper we show how to use the filter diagonalization method (FDM), a method developed and presented in several recent publications (see, e.g., refs 24-30), to obtain excited-

\footnotetext{
$\dagger$ Part of the special issue "Donald J. Kouri Festschrift".

$\doteqdot$ Heinrich-Heine-Universität Düsseldorf.

$\S$ University of California, Los Angeles.

"Hebrew University of Jerusalem.

${ }^{\perp}$ University of California, Irvine.
}

state energies from imaginary-time autocorrelation or crosscorrelation functions calculated with QMC.

While FDM is very successful in calculating excited states with real-time autocorrelation functions $\left\langle\Phi\left|\mathrm{e}^{\mathrm{i} \beta H}\right| \Phi\right\rangle$, the difficulty is in the typically very unfavorable scaling of the computational effort with the system size, similar to other accurate excitedstate methods. The imaginary-time autocorrelation function $\left\langle\Phi\left|\mathrm{e}^{-\beta H}\right| \Phi\right\rangle$ can be obtained with a quantum Monte Carlo method that scales much better with the system size.

Due to this scaling property, a QMC-based excited-state method should be particularly well suited for systems with more than a few degrees of freedom. On the other hand, it will be analyzed how the statistical noise inherent in QMC influences the accuracy of the calculated excited states.

There are two approaches for excited-state calculation within the QMC framework. In the first, and most popular, one extracts the excited-state energies from an autocorrelation function

$$
s(\beta)=\left\langle\Phi_{0}\left|\mathrm{e}^{-\beta H}\right| \Phi_{0}\right\rangle
$$

possibly using also the $H$-correlation

$$
h(\beta)=\left\langle\Phi_{0}\left|H \mathrm{e}^{-\beta H}\right| \Phi_{0}\right\rangle
$$

where $\Phi_{0}$ is an initial state with appropriate symmetry, $H$ is the full Hamiltonian, and $\beta$ is the imaginary time. Here and throughout the paper we use the non-Hermitian inner product

$$
\langle\phi \mid \psi\rangle=\langle\psi \mid \phi\rangle
$$

If $\Phi_{0}$ has a nonzero overlap with the lowest state of chosen symmetry, the resulting autocorrelation function will be dominated by the monoexponential decay $s(\beta) \sim \mathrm{e}^{-\beta E_{0}}$ for sufficiently large $\beta$, where $E_{0}$ is the corresponding eigenenergy. If $\Phi_{0}$ is made completely or approximately orthogonal to the ground state, the correlation function $s(\beta)$ would contain a large contribution from the first excited state of given symmetry. 
If the initial state $\Phi_{0}$ is dominated by several states of interest and the time signal $s(\beta)$ is computed with sufficiently high accuracy, in principle all the corresponding eigenenergies may be extracted by fitting $s(\beta)$ by the multiexponential decay

$$
s(\beta)=\sum_{k=1}^{K} d_{k} \mathrm{e}^{-\beta E_{\mathrm{k}}}
$$

Practically though a multiexponential fit cannot be carried out reliably because the corresponding inverse problem is extremely ill-conditioned, leading to very high sensitivity of $E_{k}$ to even tiny variations in $s(\beta)$. Note that this problem is closely related to the inverse Laplace transform problem, ${ }^{31}$ which has similar numerical properties. Generally for noisy data only a monoexponential fit may be carried out reliably, i.e., when $\Phi_{0}$ is dominated by a single state of interest. Specifically, for the ground-state calculation $\Phi_{0}$ could be chosen to be a Gaussian that coincides with the ground state in harmonic approximation. For a Gaussian distribution, sampling of the initial conditions for the random walks is also very efficient. For a general, e.g., excited-state, calculation the situation is much more complicated. As suggested in refs 12 and 13, one could implement an appropriate projection operator $\Phi_{0} \rightarrow A \Phi_{0}$ that projects, say a Gaussian, to the first excited state by incorporating the corresponding symmetry and/or nodal structure. However, in the case of strong anharmonicity or for a multidimensional system, such a wave function could be quite different from the true state of interest (even a small deviation in every dimension would lead to a large overall deviation). This will require solving eq 1 with large $K$, making the method impractical.

Another approach to ameliorate the problem, ${ }^{10}$ which is less often applied, aims at extracting the information from the crosscorrelation matrices

$$
\begin{gathered}
s_{\alpha \alpha^{\prime}}(\beta)=\left\langle\Phi_{\alpha}\left|\mathrm{e}^{-\beta H}\right| \Phi_{\alpha^{\prime}}\right\rangle \\
h_{\alpha \alpha^{\prime}}(\beta)=\left\langle\Phi_{\alpha}\left|H \mathrm{e}^{-\beta H}\right| \Phi_{\alpha^{\prime}}\right\rangle
\end{gathered}
$$

Here, several different initial states $\left(\Phi_{\alpha}\right)$ are used. The crosscorrelation matrices contain more information than a single correlation function; however, until recently it was not clear how to utilize this information most efficiently for the eigenenergy calculations. Therefore, applications of matrix correlation functions have generally used subspace diagonalization, a method in which the generalized eigenvalue problem associated with $s(\beta)$ and $h(\beta)$ is solved at a particular value of $\beta$ (which may then be varied for stability), i.e., not using simultaneously all the information available.

In this work we combine several methodologies mentioned above and take advantage of using the cross-correlation matrices rather than just a single correlation function, which are then processed by FDM. As we show, the use of FDM allows, for a model problem, extraction of very high quality excited-state eigenvalues.

The rest of the paper is organized as follows. First we present the methodology of the QMC calculation. Here we use the most primitive version possible, since in this publication our focus is to demonstrate the effects of FDM. Therefore, we do not use sophisticated guiding functions which would have improved the results further. Next, we review the FDM methodology, as used here. A discussion of a model problem is followed by conclusions.

\section{Computing Correlation Functions by Quantum Monte Carlo}

Usually the diffusion quantum Monte Carlo method (DQMC) is used to project an arbitrary initial state $\Phi_{0}$ to the ground state $\Psi_{0}$. However, in the present framework, we are not interested in the long-time $(\beta \rightarrow \infty)$ limit $\Psi_{0}$ but in the information generated by DQMC during short propagation time $\beta$.

For a set of initial states $\Phi_{\alpha}$ the cross-correlation functions $s_{\alpha \alpha^{\prime}}(n)$ and $h_{\alpha \alpha^{\prime}}(\beta)$ can be computed using DQMC:

$$
s_{\alpha \alpha^{\prime}}(\beta)=\left\langle\Phi_{\alpha}\left|\mathrm{e}^{-\beta H}\right| \Phi_{\alpha^{\prime}}\right\rangle=\lim _{N \rightarrow \infty} \frac{1}{N} \sum_{n=1}^{N} w_{n}(\beta) \Phi_{\alpha}\left(\mathbf{R}_{n}(\beta)\right)
$$

where $\left\{\mathbf{R}_{n}(\beta)\right\}$ denotes a sample of random walker positions at propagation time $\beta$ and walker weight $w_{n}$. The initial random walker positions $\mathbf{R}_{n}(0)$ are sampled from a distribution $p(\mathbf{R})$, and the initial weights are $w_{n}(0)=\Phi_{\alpha^{\prime}}\left(\mathbf{R}_{n}(0)\right) / p\left(\mathbf{R}_{n}(0)\right)$. As in other applications of the quantum Monte Carlo technique, the statistical accuracy of the calculated integrals is greatly increased when importance sampling is employed. ${ }^{1,32}$ Importance sampling is realized with a guide function $\Phi_{\mathrm{G}}$ and a drift step in the random walk toward large values of $\left|\Phi_{G}\right|$. Additionally, the random walk is stabilized numerically by introducing a reference energy $E_{\text {ref }} \approx E_{0}$ that shifts the energy such that the lowest eigenvalue becomes approximately zero. The propagator with importance sampling is

$$
U_{\mathrm{G}}(\beta)=\Phi_{\mathrm{G}} \mathrm{e}^{-\beta\left(H-\mathrm{E}_{\mathrm{ref}}\right)} \Phi_{\mathrm{G}}^{-1}
$$

and the cross-correlation functions can be calculated with ${ }^{10}$

$$
\begin{aligned}
s_{\alpha \alpha^{\prime}}(\beta) & =\left\langle\Phi_{\alpha} \Phi_{\mathrm{G}}{ }^{-1}\left|U_{\mathrm{G}}(\beta)\right| \Phi_{\alpha^{\prime}} \Phi_{\mathrm{G}}\right\rangle \\
& =\lim _{N \rightarrow \infty} \frac{1}{N} \sum_{n=1}^{N} w_{n}(\beta) \frac{\Phi_{\alpha}\left(\mathbf{R}_{n}(\beta)\right)}{\Phi_{\mathrm{G}}\left(\mathbf{R}_{n}(\beta)\right)}
\end{aligned}
$$

where $\left\{\mathbf{R}_{n}(\beta)\right\}$ again denotes a sample of random walker positions at propagation time $\beta$ and $w_{n}$, the corresponding weights. There are several ways to realize the importancesampled random walk, but we employ here only the simplest: the pure DQMC algorithm described by Caffarel and Claverie. ${ }^{33}$ In this algorithm, no branching is used and the weight is accumulated along the random walker trajectory at evenly sampled points $\beta=t \tau(t=0, \ldots, T)$ according to

$$
w_{n}(t \tau)=\frac{\Phi_{\alpha^{\prime}}\left(\mathbf{R}_{n}(0)\right)}{\Phi_{\mathrm{G}}\left(\mathbf{R}_{n}(0)\right)} \prod_{m=1}^{t} \exp \left\{-\frac{\tau}{\hbar}\left[E_{\mathrm{L}}\left(\mathbf{R}_{n}(m \tau)\right)-E_{\mathrm{ref}}\right]\right\}
$$

with the local energy $E_{\mathrm{L}}\left(\mathbf{R}_{n}\right)=\Phi_{\mathrm{G}}{ }^{-1}\left(\mathbf{R}_{n}\right) H \Phi_{\mathrm{G}}\left(\mathbf{R}_{n}\right)$ and the initial walker positions $\left\{\mathbf{R}_{n}(0)\right\}$ drawn from the distribution $p(\mathbf{R})$ $=\left|\Phi_{\mathrm{G}}(\mathbf{R})\right|^{2} /\left\langle\Phi_{\mathrm{G}} \mid \Phi_{\mathrm{G}}\right\rangle$. This distribution is easily obtained with a standard variational quantum Monte Carlo run ${ }^{1}$ which is based on a generalized Metropolis algorithm. ${ }^{34,35}$

Similarly we can calculate the cross-correlation functions $h_{\alpha \alpha^{\prime}}$ $(\beta)$ with

$$
\begin{aligned}
h_{\alpha \alpha^{\prime}}(\beta) & =\left\langle\Phi_{\alpha}\left|H \mathrm{e}^{-\beta H}\right| \Phi_{\alpha^{\prime}}\right\rangle \\
& =\lim _{N \rightarrow \infty} \frac{1}{N} \sum_{n=1}^{N} w_{n}(\beta) E_{\mathrm{L}}\left(\mathbf{R}_{n}(\beta)\right) \Phi_{\alpha}\left(\mathbf{R}_{n}(\beta)\right)
\end{aligned}
$$


When employing importance sampling, we obtain analogously $^{10}$

$$
\begin{aligned}
h_{\alpha \alpha^{\prime}}(\beta) & =\left\langle\Phi_{\alpha} \Phi_{\mathrm{G}}^{-1}\left|H U_{\mathrm{G}}(\beta)\right| \Phi_{\alpha^{\prime}} \Phi_{\mathrm{G}}\right\rangle \\
& =\lim _{N \rightarrow \infty} \frac{1}{N} \sum_{n=1}^{N} w_{n}(\beta) E_{\mathrm{L}}\left(\mathbf{R}_{n}(\beta)\right) \frac{\Phi_{\alpha}\left(\mathbf{R}_{n}(\beta)\right)}{\Phi_{\mathrm{G}}\left(\mathbf{R}_{n}(\beta)\right)}
\end{aligned}
$$

The use of the cross-correlation functions $s_{\alpha \alpha^{\prime}}(\beta)$ and $h_{\alpha \alpha^{\prime}}(\beta)$ with importance sampling and pure diffusion quantum Monte Carlo was proposed by Ceperley and Bernu, ${ }^{10,11}$ who used both matrices for their correlation function quantum Monte Carlo method (CFQMC) to extract excited states.

For a given sample size $N$ the statistical accuracy of $s_{\alpha \alpha^{\prime}}(\beta)$ depends on the variance of the fractions $\Phi_{\alpha}(\mathbf{R}) / \Phi_{\mathrm{G}}(\mathbf{R})$ and $\Phi_{\alpha^{\prime}-}$ $(\mathbf{R}) / \Phi_{\mathrm{G}}(\mathbf{R})$ and the variance of the local energy $E_{\mathrm{L}}(\mathbf{R})$. All three variances are small only when $\Phi_{\alpha} \approx \Phi_{\alpha^{\prime}} \approx \Phi_{\mathrm{G}}$ and $\Phi_{\mathrm{G}}$ is a good approximation to an eigenfunction of $H$. Obviously, these conditions cannot be met for many excited states. There are now two choices. One may attempt to extract a single excited state using only one function $\Phi_{\alpha}$, which approximates the excited state of interest. The guide function $\Phi_{\mathrm{G}}$ is then naturally chosen to resemble $\left|\Phi_{\alpha}\right|$. In this case only the autocorrelation functions $s_{\alpha \alpha}(\beta)$ and $h_{\alpha \alpha}(\beta)$ are used, and for each excited state a guide function needs to be constructed and a separate random walk needs to be performed.

Another possibility is to use a single guide function for all excited states and use a set of initial states that approximately span the space of the excited states of interest. Now the same random walk can be used for calculation of all the $s_{\alpha \alpha^{\prime}}$ and $h_{\alpha \alpha^{\prime}}$ matrix elements, i.e., with minimal loss of data.

\section{The Filter Diagonalization Method}

In this section we describe the H-FDM version of the filter diagonalization method designed to solve the eigenvalue problem

$$
H \mathrm{Y}_{k}=E_{k} \mathrm{Y}_{k}
$$

H-FDM differs from the previous implementations of FDM ${ }^{24-27}$ in that both cross-correlation matrices $s_{\alpha \alpha^{\prime}}(\beta)$ and $h_{\alpha \alpha^{\prime}}(\beta)$ (cf. eq 2) are used explicitly (as proposed in refs 26 and 36).

The true system Hamiltonian $H$ is Hermitian. However, because of the noise and numerical instabilities, it is not recommended to implement the hermiticity constraint, but rather treat $H$ as a non-Hermitian, but complex symmetric operator with eigenvalues, not necessarily on the real axis.

To solve eq 3 , we evaluate the matrix elements of the Hamiltonian

$$
\mathbf{H}_{\alpha j, \alpha^{\prime} j^{\prime}}:=\left\langle\Psi_{\alpha j}|H| \Psi_{\alpha^{\prime} j^{\prime}}\right\rangle
$$

and the identity operator (the overlap matrix)

$$
\mathbf{S}_{\alpha j, \alpha^{\prime} j^{\prime}}:=\left\langle\Psi_{\alpha j} \mid \Psi_{\alpha^{\prime} j^{\prime}}\right\rangle
$$

in the $z$-transformed basis:

$$
\Psi_{\alpha j} \equiv \sum_{t=0}^{M} z_{j}{ }^{-t} \mathrm{e}^{-t \tau H} \Phi_{\alpha}, \quad j=1,2, \ldots, K_{\text {win }} \text { and } \alpha=1, \ldots, L
$$

with $z_{j}$ values to be specified later.

$K_{\text {win }}$ is the number of windowing functions (see below) and $L$ the number of initial states.
The summation parameter $M$ is defined by the size, $T=2 M$, of the cross-correlation matrix $s_{\alpha \alpha^{\prime}}(t \tau),(t=0, \ldots, T)$ used in the analysis.

Due to eq 2 the needed matrix elements of $\mathbf{H}$ and $\mathbf{S}$ can be expressed solely in terms of the cross-correlation signals (see refs 24,25 , and 27 for derivation). As such for $j \neq j^{\prime}$ we have

$$
\begin{aligned}
\mathbf{S}_{\alpha j, \alpha^{\prime} j^{\prime}}= & \left(z_{j}-z_{j^{\prime}}\right)^{-1}\left[z_{j} \sum_{t=0}^{M} z_{j^{\prime}}{ }^{-t} s_{\alpha \alpha^{\prime}}(t)-z_{j^{\prime}} \sum_{t=0}^{M} z_{j}{ }^{-t} s_{\alpha \alpha^{\prime}}(t)-\right. \\
& \left.z_{j}{ }^{-M} \sum_{t=M+1}^{2 M} z_{j^{\prime}}^{M+1-t} s_{\alpha \alpha^{\prime}}(t)+z_{j^{\prime}}{ }^{-M} \sum_{t=M+1}^{2 M} z_{j}^{M+1-t} s_{\alpha \alpha^{\prime}}(t)\right]
\end{aligned}
$$

and for $j=j^{\prime}$ :

$$
\mathbf{S}_{\alpha j, \alpha^{\prime} j}=\sum_{t=0}^{2 M}(M-|M-t|+1) z_{j^{\prime}}{ }^{-t} S_{\alpha \alpha^{\prime}}(t)
$$

The elements of $\mathbf{H}$ are obtained using exactly the same formula with $s_{\alpha \alpha^{\prime}}(t)$ replaced by $h_{\alpha \alpha^{\prime}}(t)$.

Once the $\mathbf{S}$ and $\mathbf{H}$ matrices are constructed from the crosscorrelation time signals, the generalized eigenvalue problem

$$
\mathbf{H} B_{k}=\epsilon_{k} \mathbf{S} B_{k}
$$

is solved to obtain the eigenvalues $\epsilon_{k}$ that can be used to estimate the true eigenenergies $E_{k}$ (cf. eq 3 ). The total number of eigenvalues $\epsilon_{k}$ is defined by the basis size $L \times K_{\text {win }}$. In the ideal case of noiseless data and exact arithmetic, a bigger basis would give more eigenvalues and would increase their accuracy. However, in practice the number and the accuracy of the eigenvalues are limited by the very ill-conditioned nature of the problem and the fact that the time signals are noisy. Note that usually in FDM the basis is constructed by Fourier transformation of the time-dependent solution. However, here the propagation is done along the imaginary time. The choice of $\left|z_{j}\right|=1$ (i.e., on the unit circle) would then correspond to a Fourier transformation while purely real $z_{j}$ would correspond to a Laplace transformation. Most generally one can choose a set of complex numbers $z_{j}$. For the present case of nonoscillatory signals they should satisfy $z_{j} \sim 1$, the most simple choice corresponding to $K_{\text {win }}=1$ and $z_{1}=1$. The latter choice also gives the most stable results and is recommended when dealing with noisy data. Here we use the Fourier basis, i.e., $\left|z_{j}\right|=1$, and $K_{\text {win }}>1$, which would in principle result in more eigenvalues (bigger basis), but for the price of increasing numerical instability.

\section{Results}

To test the method, we used the two-dimensional (2D) harmonic oscillator as a model problem. Since the exact eigenfunctions and eigenvalues for this problem are known, and vibrational motion can often be reasonably well represented by harmonic oscillators, the $2 \mathrm{D}$ harmonic oscillator is a suitable test case for the method. A more complicated model problem such as a coupled harmonic potential would not present a more stringent test because QMC is a full many-body method that does not treat coupled potentials different from uncoupled potentials.

The Hamiltonian considered here is (in atomic units)

$$
H=-\frac{1}{2} \frac{\partial^{2}}{\partial x^{2}}-\frac{1}{2} \frac{\partial^{2}}{\partial y^{2}}+\frac{1}{2} k_{x} x^{2}+\frac{1}{2} k_{y} y^{2}
$$


with $k_{x}=1.0$ and $k_{y}=1.21$. The exact eigenvalues are

$E_{v, w}=\omega_{x}\left(v+\frac{1}{2}\right)+\omega_{y}\left(w+\frac{1}{2}\right)=$ $1.05,2.05,2.15,3.05,3.15,3.25, \ldots$

with $\omega_{x}=1.0$ and $\omega_{y}=1.1$ and $v, w=0,1, \ldots$. We chose the initial states in the form

$$
\Phi_{\alpha}=\Psi_{v, w}=H_{v}\left(\sqrt{\alpha_{x}} x\right) H_{w}\left(\sqrt{\alpha_{y}} y\right) \exp \left[-\frac{\alpha_{x}}{2} x^{2}-\frac{\alpha_{y}}{2} y^{2}\right]
$$

where the exact eigenfunctions are a special case with the parameters $\alpha_{x}=\alpha_{y}=\omega$. To simulate realistic conditions in our calculations, we intentionally used different values $\alpha_{x}$ and $\alpha_{y}$ for the initial states $\Phi_{\alpha}$.

For the guide function, we used the form suggested by Ceperley

$$
\Phi_{\mathrm{G}}=\sqrt{\sum_{\alpha=1}^{n} a_{\alpha} \Phi_{\alpha}^{2}}
$$

for which the drift term and the local energy are easily calculated from drift terms and the local energies of the basis functions.

First, we calculated the autocorrelation functions $s_{\alpha \alpha}(\beta)$ and $h_{\alpha \alpha}(\beta)$, where the initial states were $\Phi_{\alpha}=\Psi_{1,0}, \Psi_{0,1}, \Psi_{2,0}, \Psi_{1,1}$, $\Psi_{0,2}$. The time step was $\tau=0.002$. For each $\Phi_{\alpha}$ the guide function close to the corresponding initial state was used:

$$
\Phi_{\mathrm{G}}=\sqrt{0.1 \Phi_{0}^{2}+\Phi_{\alpha}{ }^{2}}
$$

The autocorrelation functions were averaged over $N=500000$ random walkers. Each pair $s_{\alpha \alpha}(\beta)$ and $h_{\alpha \alpha}(\beta)$ was processed separately by H-FDM to extract the corresponding single excited state that was well represented by the initial state. In all cases the eigenvalues obtained by the standard FDM (processing $s_{\alpha \alpha}(\beta)$ alone without explicitly using $h_{\alpha \alpha}(\beta)$ ) were noticeably less accurate, so here and throughout the paper we report only the H-FDM results.

Each H-FDM calculation was carried out using $K_{\text {win }}=10, z_{j}$

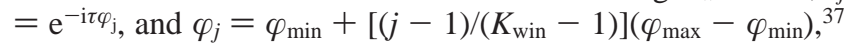
with $\varphi_{\min }=-3, \varphi_{\max }=3$, and $j=1, \ldots, K_{\text {win }}$. The resulting 10 (complex) eigenvalues $\epsilon_{k}$ were further processed to find the eigenvalue whose real part would best approximate the corresponding excited-state energy $E_{\alpha}$.

This was done by sorting the eigenvalues according to the absolute value of their imaginary parts. Starting from the smallest imaginary part, the real parts were assigned to a state using approximations $\tilde{E}_{\alpha}$ to the exact eigenstates.

The results are not very sensitive to the choice of $\varphi_{\min }$ and $\varphi_{\max }$ but depend on the basis size given by $K_{\text {win }}$. With $K_{\text {win }}=$ 1 the FDM eigenvalues are most stable and fluctuate more when $K_{\text {win }}$ is increased. However, a larger basis (e.g., $K_{\text {win }}=3$ ) gives faster convergence and higher accuracy after the eigenvalues are averaged over the processing time.

For each initial state $\Phi_{\alpha} 10$ such independent DQMC calculations of $s(\beta)$ and $h(\beta)$ were carried out, allowing us to obtain standard deviations for the calculated eigenvalues. In Figure 1 we show the results as a function of processing time $\beta$ for the lowest five excited states.

Unfortunately, the H-FDM energies fluctuate with processing time and from run to run. Such fluctuations are a manifestation of the high sensitivity of the multiexponential fit to even tiny variations in the time signal. However, because of the statistical

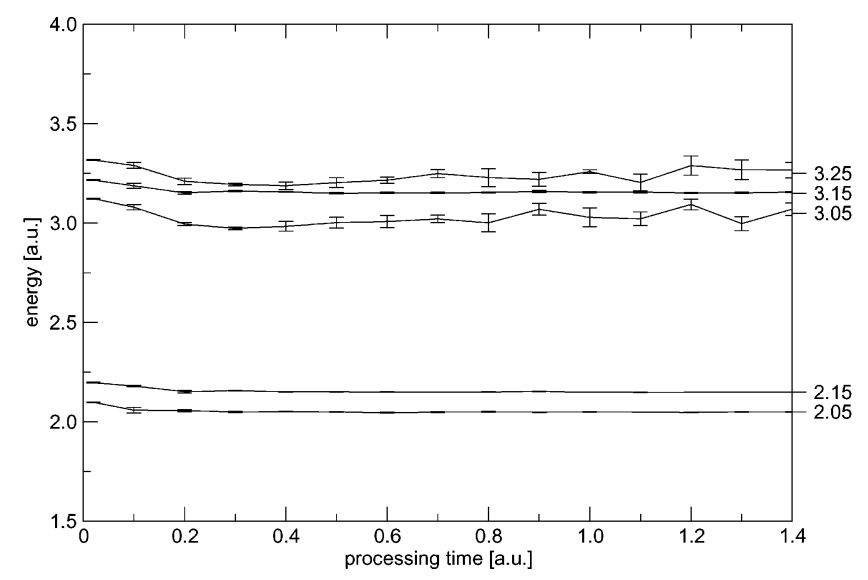

Figure 1. H-FDM results for the first five excited states of the 2D harmonic oscillator calculated from imaginary time autocorrelation functions as a function of processing time $\beta$.

TABLE 1: Excited States of 2D Harmonic Oscillator and Their Standard Deviations Calculated by H-FDM Using the Autocorrelation Functions (Single Initial State)

\begin{tabular}{cll}
\hline state & exact & \multicolumn{1}{c}{ HFDM } \\
\hline 2 & 2.05 & $2.0489 \pm 0.0004$ \\
3 & 2.15 & $2.1503 \pm 0.0007$ \\
4 & 3.05 & $3.029 \pm 0.005$ \\
5 & 3.15 & $3.1535 \pm 0.0006$ \\
6 & 3.25 & $3.243 \pm 0.006$
\end{tabular}

nature of these fluctuations, the results can be considerably improved by averaging them over independent runs and over the processing time. The latter averaging was done by discarding the data with $\beta<0.5 \mathrm{au}$. The resulting H-FDM eigenvalues are listed in Table 1. While the first two excited states are obtained accurately, the error bars of the higher excited states (except $\Psi_{1,1}$ ) are 1 order of magnitude larger. When using only the autocorrelation functions, it is difficult to improve these results further as an improvement would require either the use of better initial states or an enormous increase of the number of random walkers (to reduce the statistical noise). Furthermore, construction of each autocorrelation function requires separate DQMC calculation. However, the information accumulated by a single DQMC propagation may be used much more efficiently if the whole cross-correlation matrices are processed by H-FDM and a single guide function $\Phi_{\mathrm{G}}$ is used. In this case each random walk contributes to all the desired matrix elements $s_{\alpha \alpha^{\prime}}(\beta)$ and $h_{\alpha \alpha^{\prime}}(\beta)$.

For the cross-correlation calculation we used the same set of $L=6$ initial states $\left\{\Psi_{0,0}, \Psi_{1,0}, \Psi_{0,1}, \Psi_{2,0}, \Psi_{1,1}, \Psi_{0,2}\right\}$ with $\alpha_{x}$ $=0.8$ and $\alpha_{y}=0.9$ (denoted as "set II"). With the guide function $\Phi_{\mathrm{G}}=\sqrt{\sum_{\alpha=1}^{L} \Phi_{\alpha}{ }^{2}}$, all initial states have the same weight and all elements $s_{\alpha \alpha^{\prime}}(\beta)$ and $h_{\alpha \alpha^{\prime}}(\beta)$ are obtained from a single DQMC run using $N=500000$ random walkers.

In the H-FDM calculations, we use $K_{\text {win }}=3$ and $\varphi_{\min }=$ $-0.3, \varphi_{\max }=0.3$ for each initial state. This gives a generalized eigenvalue problem of size $L \times K_{\text {win }}=18$. Following the procedure described above for the autocorrelation functions, here six eigenvalues are selected from the list of 18 eigenvalues. (Approximate eigenenergies are readily available from a H-FDM calculation with $K_{\text {win }}=1$ and $z_{j}=1$.)

As in the case of the autocorrelation data, the results depend on the basis size determined by $K_{\text {win }}$. Increasing $K_{\text {win }}$ accelerates the convergence and the accuracy at the expense of increased numerical instability.

The eigenvalues are shown in Figure 2 as a function of processing time. The error bar is estimated from the mean square 


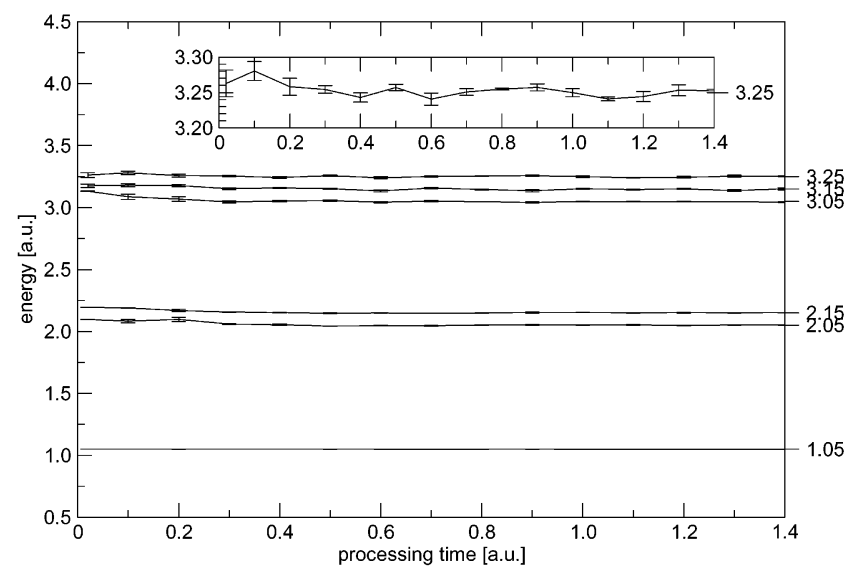

Figure 2. The lowest six eigenvalues of the 2D harmonic oscillator calculated with H-FDM using $\alpha_{x}=0.8$ and $\alpha_{y}=0.9$ and $\tau=0.002$. All results are shown with statistical error bars.

TABLE 2: Eigenvalues Calculated Simultaneously with $L=$ 6 Initial States Using Imaginary-Time H-FDM ${ }^{a}$

\begin{tabular}{ccllc}
\hline state & exact & \multicolumn{1}{c}{ set I } & \multicolumn{1}{c}{ set II } & set III \\
\hline 1 & 1.05 & $1.04993 \pm 0.00005$ & $1.04985 \pm 0.00005$ & $1.05004 \pm 0.00005$ \\
2 & 2.05 & $2.057 \pm 0.002$ & $2.0506 \pm 0.0008$ & $2.05021 \pm 0.00003$ \\
3 & 2.15 & $2.157 \pm 0.002$ & $2.1506 \pm 0.0007$ & $2.15030 \pm 0.00003$ \\
4 & 3.05 & $3.061 \pm 0.003$ & $3.0478 \pm 0.0010$ & $3.05050 \pm 0.00003$ \\
5 & 3.15 & $3.167 \pm 0.003$ & $3.1480 \pm 0.0013$ & $3.15038 \pm 0.00002$ \\
6 & 3.25 & $3.264 \pm 0.003$ & $3.2486 \pm 0.0015$ & $3.25041 \pm 0.00003$
\end{tabular}

${ }^{a}$ Sets I, II, and III have increasing quality (see text for parameters).

deviation for 10 independently calculated cross-correlation functions. The eigenvalues converge very well to the exact values after only a few time steps and are stable over a wide range of the processing time. It should be noted that the method will eventually fail at long times in the current implementation because of the well-known inherent instability of the pure diffusion Monte Carlo algorithm. ${ }^{38}$ The long-time stability of cross-correlation matrix elements can be improved with branching QMC algorithms. Here the results are stable for the entire time interval, which is partly due to the fact that in FDM the correlation function at all times contributes to the matrix elements of $\mathbf{H}$ and $\mathbf{S}$.

The present results are extremely accurate for the ground state, while for the excited states the statistical error increases slowly with quantum number. Some fluctuations of the estimated eigenvalues and their error bars can be seen in the inset of Figure 2. These fluctuations have the same origin as those seen for the autocorrelation function. Again, much better eigenvalue estimates can be obtained by averaging the results over the processing time after discarding the first few data points. For time averaging we used $\beta \geq 0.5$ au. The corresponding results (set II) are summarized in Table 2.

To check how the computed eigenvalues depend on the choice of the initial states, we repeated the calculations with the same initial states but using different parameters $\alpha_{x}=0.6$ and $\alpha_{y}=$ 0.7 (set I) and $\alpha_{x}=0.98$ and $\alpha_{y}=1.08$ (set III). Both the lowquality set (I) and the high-quality set (III) are successful in calculating all six eigenvalues with a small propagation time $\beta$ and are as stable with respect to $\beta$ as is set II. The averaged results for the other two sets are also shown in Table 2. Clearly, the statistical errors decrease when initial states of higher quality are used. For set I, at short times the cross-correlation matrix elements contain contributions from many excited states, which leads to significant overestimation of the exact results by the computed eigenvalues. For this reason the data for $\beta<0.2$ au were discarded. Nonetheless, the results obtained with the poor

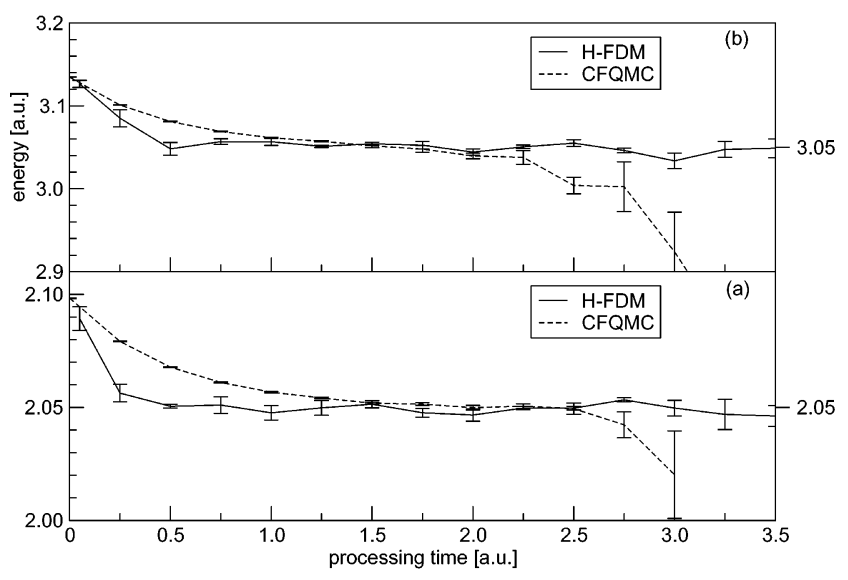

Figure 3. Comparison of H-FDM with CFQMC using the same crosscorrelation matrices $h_{\alpha \alpha^{\prime}}(\beta)$ and $s_{\alpha \alpha^{\prime}}(\beta)$. The lower panel (a) shows $E_{1,0}$, the upper panel (b) $E_{2,0}$. Set II with time step $\tau=0.005$ au was used.

set (I) are particularly encouraging as good initial states are usually unavailable for real problems.

For all three sets we observe only a moderate increase of the error bars with quantum number. In most cases the H-FDM eigenvalues overestimate the exact eigenvalues. This bias is especially obvious for the most accurate set III. Such a bias could be caused by the variational nature of the computed eigenvalues. Another reason may be the use of the finite time step $\tau$.

The H-FDM requires the same matrices $h_{\alpha \alpha^{\prime}}(\beta)$ and $s_{\alpha \alpha^{\prime}}(\beta)$ as the correlation function quantum Monte Carlo method (CFQMC). ${ }^{10} \mathrm{~A}$ direct comparison of both methods is shown in Figure 3 for two representative (excited) states. H-FDM converges for much smaller $\beta$ and is more stable for large values of $\beta$, while the CFQMC eigenvalues depend more smoothly on $\beta$ and have smaller statistical errors. The $E_{2,0}$ state demonstrates the difficulty of finding "the best" eigenvalue estimate when no plateau exists before the statistical errors increase, while the $\mathrm{H}-\mathrm{FDM}$ result is stable over the wide range of $\beta$.

\section{Conclusions}

The results presented here show the power of the H-FDM in extracting eigenvalues from the imaginary-time cross-correlation functions. The advantages of the method are due to the incorporation of both multiple spatial information (crosscorrelation between different states evolving in time) and a longrange time domain information (filter diagonalization). We have shown that the method is more stable and accurate than either the regular FDM using an autocorrelation function (i.e., no crosscorrelation) or the method of Ceperley and Bernu using the cross-correlation functions but for a particular time $\beta$ (i.e., without FDM). Notably, the cross-correlation FDM gives well converged results at very short times $(\beta \sim 0.5 \mathrm{au})$, which are significantly shorter than the inverse spacings between closely lying eigenenergies $(1 / \Delta E=10 \mathrm{au})$.

In our forthcoming publications we will apply QMC-FDM to realistic multidimensional problems, for which we anticipate the method to be equally powerful, since the QMC performance is not sensitive to the details of the Hamiltonian. The method would be ideal for studies of the lowest few excited states in large systems, such as $\mathrm{He}$ clusters and $\mathrm{He}-$ organic clusters. ${ }^{28}$

Acknowledgment. V.A.M. acknowledges support from the NSF, Grant CHE-0108823, and an Alfred P. Sloan research fellowship. D.N. acknowledges discussions with Peter Felker 
and support by the PRF and NSF. A.L. acknowledges financial support by the German Science Foundation (DFG).

\section{References and Notes}

(1) Hammond, B. L.; Lester, W. A., Jr.; Reynolds, P. J. Monte Carlo Methods in Ab Initio Quantum Chemistry; World Scientific: Singapore, 1994.

(2) Suhm, M. A.; Watts, R. O. Phys. Rep. 1991, 204, 293.

(3) Foulkes, W. M. C.; Mitas, L.; Needs, R. J.; Rajagopal, G. Rev. Mod. Phys. 2001, 73, 33-83.

(4) Anderson, J. B. J. Chem. Phys. 1976, 65, 4121.

(5) Quack, M.; Suhm, M. A. Chem. Phys. Lett. 1995, 234, 71-76.

(6) Sandler, P.; Buch, V.; Sadlej, J. J. Chem. Phys. 1996, 105, 10387.

(7) Severson, M. W.; Buch, V. J. Chem. Phys. 1999, 111, 10866.

(8) Williamson, A. J.; Hood, R. Q.; Needs, R. J.; Rajagopal, G. Phys. Rev. B 1998, 57, 12140-12144.

(9) Towler, M. D.; Hood, R. Q.; Needs, R. J. Phys. Rev. B 2000, 62, $2330-2337$.

(10) Ceperley, D. M.; Bernu, B. J. Chem. Phys. 1988, 89, 6316.

(11) Bernu, B.; Ceperley, D. M.; Lester, W. A. J. Chem. Phys. 1990, 93, 552 .

(12) Blume, D.; Lewerenz, M.; Whaley, K. B. J. Chem. Phys. 1997, $107,9067$.

(13) Blume, D.; Lewerenz, M.; Niyaz, P.; Whaley, K. B. Phys. Rev. E 1997, 55, 3664 .

(14) Prudente, F. V.; Costa, L. S.; Acioli, P. H. J. Phys. B: At. Mol. Opt. Phys. 2000, 33, R285-R313.

(15) Acioli, P. H.; Costa, L. S.; Prudente, F. V. J. Chem. Phys. 1999, 111,6311 .

(16) Acioli, P. H.; Neto, J. J. S. J. Mol. Struct. (THEOCHEM) 1999, $464,145$.

(17) da Silva, W. B.; Acioli, P. H. J. Chem. Phys. 2001, 114, 97209724.

(18) Blume, D.; Whaley, K. B. J. Chem. Phys. 2000, 112, 2218.
(19) Blume, D.; Mladenovic, M.; Lewerenz, M.; Whaley, K. B. J. Chem. Phys. 1999, 110, 5789.

(20) Cheng, E.; McMahon, M. A.; Whaley, K. B. J. Chem. Phys. 1996, 104, 2669.

(21) Kwon, Y.; Ceperley, D. M.; Whaley, K. B. J. Chem. Phys. 1996, 104, 2341.

(23) Viel, A.; Whaley, K. B. J. Chem. Phys. 2001, 115, 10186-10198.

(24) Wall, M. R.; Neuhauser, D. J. Chem. Phys. 1995, 102, 8011.

(25) Mandelshtam, V. A.; Taylor, H. S. J. Chem. Phys. 1997, 107, 6756.

(26) Narevicius, E.; Neuhauser, D.; Korsch, H. J.; Moiseyev, N. Chem. Phys. Lett. 1997, 276, 250.

(27) Mandelshtam, V. A. J. Chem. Phys. 1998, 108, 9999.

(28) Anderson, S. M.; Ka, J.; Felker, P. M.; Neuhauser, D. Chem. Phys. Lett. 2000, 328, 516

(29) Mandelshtam, V. A.; Ovchinnikov, M. J. Chem. Phys. 1998, 108, 9206.

(30) Mandelshtam, V. A. J. Phys. Chem. 2001, 105, 2764

(31) Berne, B. J. Annu. Rev. Phys. Chem. 1986, 37, 401.

(32) Freeman, D. L. Adv. Chem. Phys. 1988, 70, 139.

(33) Thirumalai, D.; Berne, B. J. Comput. Phys. Commun. 1991, 63, 415

(34) Ceperley, D. M. Rev. Mod. Phys. 1995, 67, 279. Plimak, L.; Pollak, E. J. Chem. Phys. 2000, 113, 4533.

(35) Grimm, R. C.; Storer, R. G. J. Comput. Phys. 1971, 7, 134.

(36) Caffarel, M.; Claverie, P. J. Chem. Phys. 1988, 88, 1088.

(37) Metropolis, N.; Rosenbluth, A. W.; Rosenbluth, M. N.; Teller, A. H.; Teller, E. J. Chem. Phys. 1953, 21, 1087.

(38) Kalos, M. H.; Whitlock, P. A. Monte Carlo Methods; Wiley: New York, 1986.

(39) Beck, M. H.; Meyer, H. D. J. Chem. Phys. 1998, 109, 3730

(40) Mandelshtam, V. A.; Taylor, H. S. Phys. Rev. Lett. 1997, 78, 3274.

(41) Assaraf, R.; Caffarel, M.; Khelif, A. Phys. Rev. E 2000, 61, 4566. 